\title{
SOME ANALOGUES OF A THEOREM OF PEANO FOR BOUNDARY VALUE PROBLEM
}

\author{
RICK BRANTLEY AND JOHNNY HENDERSON
}

\begin{abstract}
Under certain conditions, solutions of boundary value problems for $y^{\prime \prime \prime}=f\left(x, y, y^{\prime}, y^{\prime \prime}\right)$ are differentiated with respect to boundary conditions, both boundary points and boundary values. The results obtained are analogues of one of Peano's theorems on initial value problems.
\end{abstract}

\section{Introduction}

In this paper we will be concerned with differentiating solutions of certain boundary value problems (BVP's) with respect to both boundary values and boundary points for the third order ordinary differential equation

$$
y^{\prime \prime \prime}=f\left(x, y, y^{\prime}, y^{\prime \prime}\right)
$$

We assume that the following conditions are satisfied:

(i) $f\left(x, y_{1}, y_{2}, y_{3}\right):(a, b) \times \mathbb{R}^{3} \rightarrow \mathbb{R}$ is continuous, and

(ii) $\frac{\partial f}{\partial y_{i}}\left(x, y_{1}, y_{2}, y_{3}\right):(a, b) \times \mathbb{R}^{3} \rightarrow \mathbb{R}$ are continuous, $1 \leq i \leq 3$.

Furthermore, given a solution $y(x)$ of (1), we are also interested in solutions of BVP's for the linear third order differential equation

$$
z^{\prime \prime \prime}=\sum_{i=1}^{3} \frac{\partial f}{\partial y_{i}}\left(x, y(x), y^{\prime}(x), y^{\prime \prime}(x)\right) z^{(i-1)}
$$

Equation (2) is called the variational equation along the solution $y(x)$ of $(1)$. The variational equation along a solution $y(x)$ of the $n t h$-order differential equation,

$$
y^{(n)}=f\left(x, y, y^{\prime}, y^{\prime \prime}, \ldots, y^{(n-1)}\right)
$$

plays a fundamental role in the study of the calculus of variations [1].

Most textbooks on the theory of ordinary differential equations present a theorem addressing differentiation of solutions of (1) with respect to initial conditions. Hartman [3] attributes this well-known theorem to Peano.

Received March 19, 1990. 
In recent years, authors have obtained results that make use of differentiation of solutions of BVP's for (1) with respect to boundary conditions (both boundary points and boundary values). For example, Peterson [8]- [11], Spencer [13], and Henderson [4,5] have made use of differentiation with respect to boundary conditions and have established comparison results, obtained expressions for extremal points, and have studied some general relationships between solutions of BVP's for nonlinear ordinary differential equations and solutions of BVP's for the variational equation (2). Also, Henderson [6] proved some fairly complete analogues of the Peano Theorem for conjugate-type BVP's and right focal point BVP's. And Hankerson [2], in his dissertation, and Peterson [12] devoted some study to differentiation of solutions of finite difference equations with respect to boundary values.

In this work, we follow the path of Henderson [6] and establish complete analogues of the Peano Theorem for a family of boundary value problems for (1). Our choice of problems for this consideration is a family of boundary value problems for (1) that have been studied extensively by Henderson [5] for uniqueness, existence, and optimality.

In Section 2, we state Peano's Theorem for (1). We then list the boundary value problems for (1) for which we are interested in proving analogues of Peano's Theorem. The proofs of these analogues for boundary value problems depend first on uniqueness of solutions conditions, which we enumerate as additional assumptions on (1).

In Section 3, for one of our boundary value problems, we state, without proof, a theorem concerning continuity of solutions with respect to boundary conditions. We then prove a theorem concerning differentiation of solutions, for that boundary value problem, with respect to boundary conditions (both boundary points and boundary values), which is a complete analogue of Peano's Theorem.

In Section 4, we state, without proof, a couple of theorems concerning differentiation of solutions with respect to boundary conditions along with corresponding theorems on continuous dependence of solutions with respect to boundary conditions.

\section{A Theorem by Peano}

We begin by stating a theorem due to Peano for initial value problems.

Theorem 2.1 (Peano) Let $x_{0} \in(a, b)$ and $y(x) \equiv y\left(x ; x_{0}, c_{1}, c_{2}, c_{3}\right)$ denote the solution of (1) satisfying the initial conditions $y\left(x_{0}\right)=c_{1}, y^{\prime}\left(x_{0}\right)=c_{2}$, and $y^{\prime \prime}\left(x_{0}\right)=c_{3}$ and let $(\alpha, \omega) \subseteq(a, b)$ be the maximal interval of existence of $y(x)$. Then

(a) $\frac{\partial y}{\partial c_{1}}, \frac{\partial y}{\partial c_{2}}, \frac{\partial y}{\partial c_{3}}$ exist on $(\alpha, \omega)$ and $\alpha_{i} \equiv \frac{\partial y}{\partial c_{i}}, 1 \leq i \leq 3$, are solutions of the variational equation (2) along $y(x)$ satisfying the initial conditions

$$
\begin{aligned}
& \alpha_{1}\left(x_{0}\right)=1, \alpha_{1}^{\prime}\left(x_{0}\right)=0, \alpha_{1}^{\prime \prime}\left(x_{0}\right)=0, \\
& \alpha_{2}\left(x_{0}\right)=0, \alpha_{2}^{\prime}\left(x_{0}\right)=1, \alpha_{2}^{\prime \prime}\left(x_{0}\right)=0, \\
& \alpha_{3}\left(x_{0}\right)=0, \alpha_{3}^{\prime}\left(x_{0}\right)=0, \alpha_{3}^{\prime \prime}\left(x_{0}\right)=1 .
\end{aligned}
$$


(b) $\frac{\partial y}{\partial x_{0}}$ exists on $(\alpha, \omega)$ and $\beta \equiv \frac{\partial y}{\partial x_{0}}$ is the solution of the variational equation (2) along $y(x)$ satisfying the initial conditions

$$
\beta\left(x_{0}\right)=-y^{\prime}\left(x_{0}\right), \quad \beta^{\prime}\left(x_{0}\right)=-y^{\prime \prime}\left(x_{0}\right), \quad \beta^{\prime \prime}\left(x_{0}\right)=-y^{\prime \prime \prime}\left(x_{0}\right) .
$$

(c) $\frac{\partial y}{\partial x_{0}}(x)=-y^{\prime}\left(x_{0}\right) \frac{\partial y}{\partial c_{1}}(x)-y^{\prime \prime}\left(x_{0}\right) \frac{\partial y}{\partial c_{2}}(x)-y^{\prime \prime \prime}\left(x_{0}\right) \frac{\partial y}{\partial c_{3}}(x)$.

With respect to the third-order differential equation (1), we will be concerned with analogues of Theorem 2.1 for BVP's satisfying the following types of boundary conditions:

$$
\begin{aligned}
& y\left(x_{1}\right)=y_{1}, y^{\prime}\left(x_{2}\right)=y_{2}, y^{\prime}\left(x_{3}\right)=y_{3}, \quad a<x_{1}<x_{2}<x_{3}<b, \\
& y\left(x_{1}\right)=y_{1}, y^{\prime}\left(x_{1}\right)=y_{2}, y^{\prime}\left(x_{2}\right)=y_{3}, \quad a<x_{1}<x_{2}<b, \\
& y\left(x_{1}\right)=y_{1}, y^{\prime}\left(x_{2}\right)=y_{2}, y^{\prime \prime}\left(x_{2}\right)=y_{3}, \quad a<x_{1}<x_{2}<b .
\end{aligned}
$$

Remark. Henderson [5] has studied BVP's for (1) satisfying the above types of conditions for uniqueness, existence, and optimality.

Our analogues will depend on uniqueness assumptions for each respective boundary value problem above. Specifically, we will have need of the following assumptions:

(iii) Given $a<x_{1}<x_{2}<x_{3}<b$, if $y\left(x_{1}\right)=z\left(x_{1}\right), y^{\prime}\left(x_{2}\right)=z^{\prime}\left(x_{2}\right)$, and $y^{\prime}\left(x_{3}\right)=z^{\prime}\left(x_{3}\right)$, where $y(x)$ and $z(x)$ are solutions of $(1)$, then $y(x) \equiv z(x)$ on $\left[x_{1}, x_{3}\right]$.

(iv) Given $a<x_{1}<x_{2}<b$, if $y\left(x_{1}\right)=z\left(x_{1}\right), y^{\prime}\left(x_{1}\right)=z^{\prime}\left(x_{1}\right)$, and $y^{\prime}\left(x_{2}\right)=z^{\prime}\left(x_{2}\right)$, where $y(x)$ and $z(x)$ are solutions of $(1)$, then $y(x) \equiv z(x)$ on $\left[x_{1}, x_{2}\right]$.

(v) Given $a<x_{1}<x_{2}<b$, if $y\left(x_{1}\right)=z\left(x_{1}\right), y^{\prime}\left(x_{2}\right)=z^{\prime}\left(x_{2}\right)$, and $y^{\prime \prime}\left(x_{2}\right)=z^{\prime \prime}\left(x_{2}\right)$, where $y(x)$ and $z(x)$ are solutions of $(1)$, then $y(x) \equiv z(x)$ on $\left[x_{1}, x_{2}\right]$. of (1):

We will also have need of similar uniqueness assumptions on (2) along solutions $y(x)$

(iii') Given $a<x_{1}<x_{2}<x_{3}<b$, and a solution $y(x)$ of (1), if $u\left(x_{1}\right)=0, u^{\prime}\left(x_{2}\right)=0$, and $u^{\prime}\left(x_{3}\right)=0$, where $u(x)$ is a solution of (2) along $y(x)$, then $u(x) \equiv 0$ on $\left[x_{1}, x_{3}\right]$.

(iv') Given $a<x_{1}<x_{2}<b$, and a solution $y(x)$ of $(1)$, if $u\left(x_{1}\right)=0, u^{\prime}\left(x_{1}\right)=0$, and $u^{\prime}\left(x_{2}\right)=0$, where $u(x)$ is a solution of (2) along $y(x)$, then $u(x) \equiv 0$ on $\left[x_{1}, x_{2}\right]$.

$\left(\mathrm{v}^{\prime}\right)$ Given $a<x_{1}<x_{2}<b$, and a solution $y(x)$ of $(1)$, if $u\left(x_{1}\right)=0, u^{\prime}\left(x_{2}\right)=0$, and $u^{\prime \prime}\left(x_{2}\right)=0$, where $u(x)$ is a solution of (2) along $y(x)$, then $u(x) \equiv 0$ on $\left[x_{1}, x_{2}\right]$.

\section{An Analogue of Peamo's Theorem for $\mathbb{B V P}$ 's}

We now prove an analogue of Peano's Theorem for the BVP (1), (3) in the spirit of results obtained by Henderson [6]. In order to do this, it first must the case that solutions depend continuously on boundary conditions. So, we begin by stating, without proof, such a theorem on continuous dependence. The proof of this theorem would follow along the lines of the one given in [7].

Theorem 3.1. Assume (i), (ii), and (iii) are satisfied with respect to (1). Let $u(x)$ be a solution of (1) with maximal interval of existence $(\alpha, \omega) \subseteq(a, b)$ and let $\alpha<x_{0}<$ 
$x_{1}<x_{2}<x_{3}<x_{4}<\omega$ be given. Then there exists a $\delta>0$ such that, for $\left|x_{1}-t_{1}\right|<$ $\delta,\left|x_{2}-t_{2}\right|<\delta,\left|x_{3}-t_{3}\right|<\delta,\left|u\left(x_{1}\right)-y_{1}\right|<\delta,\left|u^{\prime}\left(x_{2}\right)-y_{2}\right|<\delta$, and $\left|u^{\prime}\left(x_{3}\right)-y_{3}\right|<\delta$, there is a unique solution $u_{\delta}(x)$ of $(1)$ whose maximal interval of existence is $\left(\alpha_{\delta}, \omega_{\delta}\right) \supset\left[x_{0}, x_{4}\right]$ and is such that $u_{\delta}\left(t_{1}\right)=y_{1}, u_{\delta}^{\prime}\left(t_{2}\right)=y_{2}, u_{\delta}^{\prime}\left(t_{3}\right)=y_{3}$. Further, $\left\{u_{\delta}^{(j)}(x)\right\}$ converges uniformly to $u^{(j)}(x)$, as $\delta \rightarrow 0$, on $\left[x_{0}, x_{4}\right]$, for each $j=0,1,2$.

Theorem 3.2. Assume (i), (ii), and (iii) are satisfied. Also assume that the variational equation (2), along any solution $y(x)$ of (1) satisfies $\left(\right.$ iii $\left.i^{\prime}\right)$ on $(a, b)$. Let $u(x)$ be a solution of (1) with maximal interval of existence $(\alpha, \omega) \subseteq(a, b)$. Let $\alpha<x_{1}<x_{2}<$ $x_{3}<\omega$ be given, so that $u(x)=u\left(x ; x_{1}, x_{2}, x_{3}, u_{1}, u_{2}, u_{3}\right)$, where $u\left(x_{1}\right)=u_{1}, u^{\prime}\left(x_{2}\right)=u_{2}$, and $u^{\prime}\left(x_{3}\right)=u_{3}$. Then

(a) $\frac{\partial u}{\partial u_{1}}, \frac{\partial u}{\partial u_{2}}, \frac{\partial u}{\partial u_{3}}$ exist on $(\alpha, \omega)$ and $y_{i} \equiv \frac{\partial u}{\partial u_{i}}, 1 \leq i \leq 3$, is the solution of (2) along $u(x)$ and satisfies the boundary conditions

$$
\begin{aligned}
& y_{1}\left(x_{1}\right)=1, y_{1}^{\prime}\left(x_{2}\right)=0, y_{1}^{\prime}\left(x_{3}\right)=0, \\
& y_{2}\left(x_{1}\right)=0, y_{2}^{\prime}\left(x_{2}\right)=1, y_{2}^{\prime}\left(x_{3}\right)=0, \\
& y_{3}\left(x_{1}\right)=0, y_{3}^{\prime}\left(x_{2}\right)=0, y_{3}^{\prime}\left(x_{3}\right)=1 .
\end{aligned}
$$

(b) $\frac{\partial u}{\partial x_{1}}, \frac{\partial u}{\partial x_{2}}, \frac{\partial u}{\partial x_{3}}$ exist on $(\alpha, \omega)$ and $z_{i} \equiv \frac{\partial u}{\partial x_{i}}, 1 \leq i \leq 3$, is the solution of (2) along $u(x)$ and satisfies the boundary conditions

$$
\begin{aligned}
z_{1}\left(x_{1}\right)=-u^{\prime}\left(x_{1}\right), z_{1}^{\prime}\left(x_{2}\right)=0, z_{1}^{\prime}\left(x_{3}\right) & =0, \\
z_{2}\left(x_{1}\right)=0, z_{2}^{\prime}\left(x_{2}\right)=-u^{\prime \prime}\left(x_{2}\right), z_{2}^{\prime}\left(x_{3}\right) & =0, \\
z_{3}\left(x_{1}\right)=0, z_{3}^{\prime}\left(x_{2}\right)=0, z_{3}^{\prime}\left(x_{3}\right) & =-u^{\prime \prime}\left(x_{3}\right) .
\end{aligned}
$$

(c) The partial derivatives satisfy

$$
\begin{aligned}
& \frac{\partial}{\partial x_{1}} u(x)=-u^{\prime}\left(x_{1}\right) \frac{\partial}{\partial u_{1}} u(x), \\
& \frac{\partial}{\partial x_{2}} u(x)=-u^{\prime \prime}\left(x_{2}\right) \frac{\partial}{\partial u_{2}} u(x), \\
& \frac{\partial}{\partial x_{3}} u(x)=-u^{\prime \prime}\left(x_{3}\right) \frac{\partial}{\partial u_{3}}(x) .
\end{aligned}
$$

Proof. For part (a) we will give the argument for $\frac{\partial u}{\partial u_{1}}$, since the arguments for $\frac{\partial u}{\partial u_{2}}$ and $\frac{\partial u}{\partial u_{3}}$ are similar. Let $\delta>0$ be as in Theorem 3.1. Let $0<|h|<\delta$ be given and define

$$
y_{1 h}(x)=\frac{u\left(x ; x_{1}, x_{2}, x_{3}, u_{1}+h, u_{2}, u_{3}\right)-u\left(x ; x_{1}, x_{2}, x_{3}, u_{1}, u_{2}, u_{3}\right)}{h}
$$

Note that $u\left(x_{1} ; x_{1}, x_{2}, x_{3}, u_{1}+h, u_{2}, u_{3}\right)=u_{1}+h$, and $u\left(x_{1} ; x_{1}, x_{2}, x_{3}, u_{1}, u_{2}, u_{3}\right)=u_{1}$, so that

$$
y_{1 h}\left(x_{1}\right)=\frac{u_{1}+h-u_{1}}{h}=1, \quad y_{1 h}^{\prime}\left(x_{2}\right)=\frac{u_{2}-u_{2}}{h}=0, \text { and } y_{1 h}^{\prime}\left(x_{3}\right)=\frac{u_{3}-u_{3}}{h}=0
$$


for every $h \neq 0$.

Let

$$
\begin{aligned}
\beta_{2} & =u^{\prime}\left(x_{1} ; x_{1}, x_{2}, x_{3}, u_{1}, u_{2}, u_{3}\right), \beta_{3}=u^{\prime \prime}\left(x_{1} ; x_{1}, x_{2}, x_{3}, u_{1}, u_{2}, u_{3}\right), \\
\epsilon_{2} & =\epsilon_{2}(h)=u^{\prime}\left(x_{1} ; x_{1}, x_{2}, x_{3}, u_{1}+h, u_{2}, u_{3}\right)-\beta_{2}, \\
\text { and } \epsilon_{3} & =\epsilon_{3}(h)=u^{\prime \prime}\left(x_{1} ; x_{1}, x_{2}, x_{3}, u_{1}+h, u_{2}, u_{3}\right)-\beta_{3} .
\end{aligned}
$$

By Theorem 3.1, $\epsilon_{2}=\epsilon_{2}(h) \rightarrow 0$ and $\epsilon_{3}=\epsilon_{3}(h) \rightarrow 0$, as $h \rightarrow 0$.

For notational purposes, given $p \in(a, b)$ and $c_{1}, c_{2}, c_{3} \in \mathbb{R}$, let $y\left(x ; p, c_{1}, c_{2}, c_{3}\right)$ denote the solution of (1) satisfying the initial conditions

$$
y(p)=c_{1}, y^{\prime}(p)=c_{2}, y^{\prime \prime}(p)=c_{3} .
$$

Then by viewing the solutions $u$ as solutions of initial value problems and using a telescoping sum, we have

$$
\begin{aligned}
y_{1 h}(x)= & \frac{1}{h}\left\{u\left(x ; x_{1}, x_{2}, x_{3}, u_{1}+h, u_{2}, u_{3}\right)-u\left(x ; x_{1}, x_{2}, x_{3}, u_{1}, u_{2}, u_{3}\right)\right\} \\
= & \frac{1}{h}\left\{y\left(x ; x_{1}, u_{1}, u_{1}+h, \beta_{2}+\epsilon_{2}, \beta_{3}+\epsilon_{3}\right)-y\left(x ; x_{1}, u_{1}, \beta_{2}, \beta_{3}\right)\right\} \\
= & \frac{1}{h}\left\{\left[y\left(x ; x_{1}, u_{1}+h, \beta_{2}+\epsilon_{2}, \beta_{3}+\epsilon_{3}\right)-y\left(x ; x_{1}, u_{1}, \beta_{2}+\epsilon_{2}, \beta_{3}+\epsilon_{3}\right)\right]\right. \\
& +\left[y\left(x ; x_{1}, u_{1}, \beta_{2}+\epsilon_{2}, \beta_{3}+\epsilon_{3}\right)-y\left(x ; x_{1}, u_{1}, \beta_{2}, \beta_{3}+\epsilon_{3}\right)\right] \\
& \left.+\left[y\left(x ; x_{1}, u_{1}, \beta_{2}, \beta_{3}+\epsilon_{3}\right)-y\left(x ; x_{1}, u_{1}, \beta_{2}, \beta_{3}\right)\right]\right\} .
\end{aligned}
$$

By Theorem 2.1 and the Mean Value Theorem, we obtain

$$
\begin{aligned}
y_{1 h}(x)= & \frac{1}{h} \alpha_{1}\left(x ; y\left(x ; x_{1}, u_{1}+\bar{h}, \beta_{2}+\epsilon_{2}, \beta_{3}+\epsilon_{3}\right)\right)\left(u_{1}+h-u_{1}\right) \\
& +\frac{1}{h} \alpha_{2}\left(x ; y\left(x ; x_{1}, u_{1}, \beta_{2}+\bar{\epsilon}_{2}, \beta_{3}+\epsilon_{3}\right)\right)\left(\beta_{2}+\epsilon_{2}-\beta_{2}\right) \\
& +\frac{1}{h} \alpha_{3}\left(x ; y\left(x ; x_{1}, u_{1}, \beta_{2}, \beta_{3}+\bar{\epsilon}_{3}\right)\right)\left(\beta_{3}+\epsilon_{3}-\beta_{3}\right),
\end{aligned}
$$

where $\alpha_{i}(x ; y(\cdot)), 1 \leq i \leq 3$, is the solution of the variational equation (2) along $y(\cdot)$ and satisfies in each case

$$
\begin{aligned}
& \alpha_{1}\left(x_{1}\right)=1, \alpha_{1}^{\prime}\left(x_{1}\right)=0, \alpha_{1}^{\prime \prime}\left(x_{1}\right)=0, \\
& \alpha_{2}\left(x_{1}\right)=0, \alpha_{2}^{\prime}\left(x_{1}\right)=1, \alpha_{2}^{\prime \prime}\left(x_{1}\right)=0, \\
& \alpha_{3}\left(x_{1}\right)=0, \alpha_{3}^{\prime}\left(x_{1}\right)=0, \alpha_{3}^{\prime \prime}\left(x_{1}\right)=1 .
\end{aligned}
$$

Furthermore, $u_{1}+\bar{h}$ is between $u_{1}$ and $u_{1}+h, \beta_{2}+\bar{\epsilon}_{2}$ is between $\beta_{2}$ and $\beta_{2}+\epsilon_{2}$, and $\beta_{3}+\bar{\epsilon}_{3}$ is between $\beta_{3}$ and $\beta_{3}+\epsilon_{3}$. Now simplyifying,

$$
\begin{aligned}
y_{1 h}(x)= & \alpha_{1}\left(x ; y\left(x ; x_{1}, u_{1}+\bar{h}, \beta_{2}+\epsilon_{2}, \beta_{3}+\epsilon_{3}\right)\right) \\
& +\frac{\epsilon_{2}}{h} \alpha_{2}\left(x ; y\left(x ; x_{1}, u_{1}, \beta_{2}+\overline{\epsilon_{2}}, \beta_{3}+\epsilon_{3}\right)\right) \\
& +\frac{\epsilon_{3}}{h} \alpha_{3}\left(x ; y\left(x ; x_{1}, u_{1}, \beta_{2}, \beta_{3}+\bar{\epsilon}_{3}\right)\right) .
\end{aligned}
$$


Thus, to show that $\lim _{h \rightarrow 0} y_{1 h}(x)$ exists, we show that $\lim _{h \rightarrow 0} \frac{\epsilon_{2}}{h}$ exists, and $\lim _{h \rightarrow 0} \frac{\epsilon_{3}}{h}$ exists.

Recalling that $y_{1 h}^{\prime}\left(x_{2}\right)=y_{1 h}^{\prime}\left(x_{3}\right)=0$, we can write

$$
\begin{aligned}
& -\alpha_{1}^{\prime}\left(x_{2} ; y\left(x ; x_{1}, u_{1}+\bar{h}, \beta_{2}+\epsilon_{2}, \beta_{3}+\epsilon_{3}\right)\right) \\
= & \frac{\epsilon_{2}}{h} \alpha_{2}^{\prime}\left(x_{2} ; y\left(x ; x_{1}, u_{1}, \beta_{2}+\bar{\epsilon}_{2}, \beta_{3}+\epsilon_{3}\right)\right) \\
& +\frac{\epsilon_{3}}{h} \alpha_{3}^{\prime}\left(x_{2} ; y\left(x ; x_{1}, u_{1}, \beta_{2}, \beta_{3}+\bar{\epsilon}_{3}\right)\right),
\end{aligned}
$$

and

$$
\begin{aligned}
& -\alpha_{1}^{\prime}\left(x_{3} ; y\left(x ; x_{1}, u_{1}+\bar{h}, \beta_{2}+\epsilon_{2}, \beta_{3}+\epsilon_{3}\right)\right) \\
= & \frac{\epsilon_{2}}{h} \alpha_{2}^{\prime}\left(x_{3} ; y\left(x ; x_{1}, u_{1}, \beta_{2}+\bar{\epsilon}_{2}, \beta_{3}+\epsilon_{3}\right)\right) \\
& +\frac{\epsilon_{3}}{h} \alpha_{3}^{\prime}\left(x_{3} ; y\left(x ; x_{1}, u_{1}, \beta_{2}, \beta_{3}+\bar{\epsilon}_{3}\right)\right) .
\end{aligned}
$$

This gives us a system of two equations in the two unkonwns $\frac{\epsilon_{2}}{h}$ and $\frac{\epsilon_{3}}{h}$. By Cramer's Rule

provided

$$
\begin{gathered}
\frac{\epsilon_{2}}{h}=\frac{\left|\begin{array}{ll}
-\alpha_{1}^{\prime}\left(x_{2} ; y(\cdot)\right) & \alpha_{3}^{\prime}\left(x_{2} ; y(\cdot)\right) \\
-\alpha_{1}^{\prime}\left(x_{3} ; y(\cdot)\right) & \alpha_{3}^{\prime}\left(x_{3} ; y(\cdot)\right)
\end{array}\right|}{D(h)} \equiv \frac{D_{2}(h)}{D(h)}, \\
\frac{\epsilon_{3}}{h}=\frac{\left|\begin{array}{ll}
\alpha_{2}^{\prime}\left(x_{2} ; y(\cdot)\right) & -\alpha_{1}^{\prime}\left(x_{2} ; y(\cdot)\right) \\
\alpha_{2}^{\prime}\left(x_{3} ; y(\cdot)\right) & -\alpha_{1}^{\prime}\left(x_{3} ; y(\cdot)\right)
\end{array}\right|}{D(h)} \equiv \frac{D_{3}(h)}{D(h)}
\end{gathered}
$$

$$
\begin{aligned}
& 0 \neq D(h) \equiv \\
& \quad\left|\begin{array}{ll}
\alpha_{2}^{\prime}\left(x_{2} ; y\left(x ; x_{1}, u_{1}, \beta_{2}+\bar{\epsilon}_{2}, \beta_{3}+\epsilon_{3}\right)\right) & \alpha_{3}^{\prime}\left(x_{2} ; y\left(x ; x_{1}, u_{1}, \beta_{2}, \beta_{3}+\bar{\epsilon}_{3}\right)\right) \\
\alpha_{2}^{\prime}\left(x_{3} ; y\left(x ; x_{1}, u_{1}, \beta_{2}+\bar{\epsilon}_{2}, \beta_{3}+\epsilon_{3}\right)\right) & \alpha_{3}^{\prime}\left(x_{3} ; y\left(x ; x_{1}, u_{1}, \beta_{2}, \beta_{3}+\bar{\epsilon}_{3}\right)\right)
\end{array}\right| .
\end{aligned}
$$

Now $\alpha_{2}\left(x ; y\left(x ; x_{1}, u_{1}, \beta_{2}, \beta_{3}\right)\right)$ and $\alpha_{3}\left(x ; y\left(x ; x_{1}, u_{1}, \beta_{2}, \beta_{3}\right)\right)$ are both zero at $x=x_{1}$. We claim that no nontrivial combination $A \alpha_{2}\left(x ; y\left(x ; x_{1}, u_{1}, \beta_{2}, \beta_{3}\right)\right)+B \alpha_{3}\left(x ; y\left(x ; x_{1}, u_{1}, \beta_{2}\right.\right.$, $\left.\beta_{3}\right)$ ) has derivative that vanishes at both $x=x_{2}$ and $x=x_{3}$. To see this, suppose $A \alpha_{2}(x ; y(x))+B \alpha_{3}(x ; y(x))$ has derivative equal to zero at $x=x_{2}$ and $x=x_{3}$ and that not both $A$ and $B$ are zero. Then by condition (iii'), $A \alpha_{2}(x ; y(x))+B \alpha_{3}(x ; y(x)) \equiv 0$.

Now, since $\alpha_{2}(x ; y(x))$ and $\alpha_{3}(x ; y(x))$ are nontrivial solutions of $(2)$, then $\alpha_{2}(x ; y(x))$ $=k \alpha_{3}(x ; y(x))$, for some $k \neq 0$. But $\alpha_{2}^{\prime}\left(x_{1} ; y(x)\right)=1, k \alpha_{3}^{\prime}\left(x_{1} ; y(x)\right)=k \cdot 0$, which is a contradiction. So there is no nontrivial linear combination $A \alpha_{2}(x ; y(x))+B \alpha_{3}(x ; y(x))$ that has derivative equal to zero at $x=x_{2}$ and $x=x_{3}$. This is equivalent to

$$
0 \neq D=\left|\begin{array}{cc}
\alpha_{2}^{\prime}\left(x_{2} ; y\left(x ; x_{1}, u_{1}, \beta_{2}, \beta_{3}\right)\right) & \alpha_{3}^{\prime}\left(x_{2} ; y\left(x ; x_{1}, u_{1}, \beta_{2}, \beta_{3}\right)\right) \\
\alpha_{2}^{\prime}\left(x_{3} ; y\left(x ; x_{1}, u_{1}, \beta_{2}, \beta_{3}\right)\right) & \alpha_{3}^{\prime}\left(x_{3} ; y\left(x ; x_{1}, u_{1}, \beta_{2}, \beta_{3}\right)\right)
\end{array}\right|
$$


And by Theorem 3.1, $D(h) \neq 0$ for $h$ sufficiently small. In fact, $\lim _{h \rightarrow 0} D(h)=D$.

Hence, taking limits, we have

$$
\lim _{h \rightarrow 0} \frac{\epsilon_{2}}{h}=\lim _{h \rightarrow 0} \frac{D_{2}(h)}{D(h)} \equiv \frac{D_{2}}{D} \equiv \text { constant, and } \lim _{h \rightarrow 0} \frac{\epsilon_{3}}{h} \frac{D_{3}(h)}{D(h)} \equiv \frac{D_{3}}{D} \equiv \text { constant } .
$$

As a result of this,

$$
\begin{aligned}
y_{1 h}(x)= & \alpha_{1}\left(x ; y\left(x ; x_{1}, u_{1}+\bar{h}, \beta_{2}+\epsilon_{2}, \beta_{3}+\epsilon_{3}\right)\right) \\
& +\frac{D_{2}(h)}{D(h)} \alpha_{2}\left(x ; y\left(x ; x_{1}, u_{1}, \beta_{2}+\bar{\epsilon}_{2}, \beta_{3}+\epsilon_{3}\right)\right) \\
& +\frac{D_{3}(h)}{D(h)} \alpha_{3}\left(x ; y\left(x ; x_{1}, u_{1}, \beta_{2}, \beta_{3}+\bar{\epsilon}_{3}\right)\right),
\end{aligned}
$$

and $\lim _{h \rightarrow 0} y_{1 h}(x)$ exists since the limit on the righthand side of the equation exists. Let $y_{1}(x)=\lim _{h \rightarrow 0} y_{1 h}(x)$. Note that by the construction of $y_{1 h}(x)$,

$$
y_{1}(x)=\frac{\partial u}{\partial u_{1}}\left(x ; x_{1}, x_{2}, x_{3}, u_{1}, u_{2}, u_{3}\right)
$$

In particular, we now have the existence of $\frac{\partial u}{\partial u_{1}}$. Furthermore,

$$
\begin{aligned}
y_{1}(x)= & \lim _{h \rightarrow 0} y_{1 h}(x) \\
= & \lim _{h \rightarrow 0} \alpha_{1}\left(x ; y\left(x ; x_{1}, u_{1}+\bar{h}, \beta_{2}+\epsilon_{2}, \beta_{3}+\epsilon_{3}\right)\right) \\
& +\lim _{h \rightarrow 0} \frac{D_{2}(h)}{D(h)} \alpha_{2}\left(x ; y\left(x ; x_{1}, u_{1}, \beta_{2}+\bar{\epsilon}_{2}, \beta_{3}+\epsilon_{3}\right)\right) \\
& +\lim _{h \rightarrow 0} \frac{D_{3}(h)}{D(h)} \alpha_{3}\left(x ; y\left(x ; x_{1}, u_{1}, \beta_{2}, \beta_{3}+\bar{\epsilon}_{3}\right)\right),
\end{aligned}
$$

which implies that

$$
\begin{aligned}
y_{1}(x)= & \alpha_{1}\left(x ; y\left(x ; x_{1}, u_{1}, \beta_{2}, \beta_{3}\right)\right) \\
& +\frac{D_{2}}{D} \alpha_{2}\left(x ; y\left(x ; x_{1}, u_{1}, \beta_{2}, \beta_{3}\right)\right) \\
& +\frac{D_{3}}{D} \alpha_{3}\left(x ; y\left(x ; x_{1}, u_{1}, \beta_{2}, \beta_{3}\right)\right) .
\end{aligned}
$$

So, $y_{1}(x)$ is the solution of (2) along $y\left(x ; x_{1}, u_{1}, \beta_{2}, \beta_{3}\right)=u\left(x ; x_{1}, x_{2}, x_{3}, u_{1}, u_{2}, u_{3}\right)$, since $\alpha_{1}(x), \alpha_{2}(x), \alpha_{3}(x)$ are all solutions of the linear homogeneous variational equation (2) along $u(x)$.

For the boundary conditions, first

$$
y_{1}\left(x_{1}\right)=\lim _{h \rightarrow 0} y_{1 h}\left(x_{1}\right)=\lim _{h \rightarrow 0} 1=1 .
$$


And from the beginning part of this proof, we also have

$$
\begin{aligned}
& y_{1}^{\prime}\left(x_{2}\right)=\lim _{h \rightarrow 0} y_{1 h}^{\prime}\left(x_{2}\right)=\lim _{h \rightarrow 0} 0=0, \text { and } \\
& y_{1}^{\prime}\left(x_{3}\right)=\lim _{h \rightarrow 0} y_{1 h}^{\prime}\left(x_{3}\right)=\lim _{h \rightarrow 0} 0=0 .
\end{aligned}
$$

This completes the argument for $\frac{\partial u}{\partial u_{1}}$.

In part (b) of the theorem, we will produce the details for $\frac{\partial u}{\partial x_{1}}$ and $\frac{\partial u}{\partial x_{2}}$ and omit the argument for $\frac{\partial u}{\partial x_{3}}$, since it is similar to $\frac{\partial u}{\partial x_{2}}$. First, we show the existence of $\frac{\partial u}{\partial x_{1}}$. Let $\delta>0$ be given as in Theorem 3.1 , choose $h \neq 0$ with $|h|<\delta$, and define

$$
z_{1 h}(x)=\frac{u\left(x ; x_{1}+h, x_{2}, x_{3}, u_{1}, u_{2}, u_{3}\right)-u\left(x ; x_{1}, x_{2}, x_{3}, u_{1}, u_{2}, u_{3}\right)}{h} .
$$

Note that $z_{1 h}^{\prime}\left(x_{2}\right)=\frac{u_{2}-u_{2}}{h}=0$, for every $h \neq 0$, and $z_{1 h}^{\prime}\left(x_{3}\right)=\frac{u_{3}-u_{3}}{h}=0$, for every $h \neq 0$.

Let $\beta_{2}=u^{\prime}\left(x_{1} ; x_{1}, x_{2}, x_{3}, u_{1}, u_{2}, u_{3}\right)$, and

$$
\beta_{3}=u^{\prime \prime}\left(x_{1} ; x_{1}, x_{2}, x_{3}, u_{1}, u_{2}, u_{3}\right) \text {. }
$$

Remark. $u_{1}=u\left(x_{1} ; x_{1}, x_{2}, x_{3}, u_{1}, u_{2}, u_{3}\right)$

$$
\text { and } u_{1}=u\left(x_{1}+h ; x_{1}+h, x_{2}, x_{3}, u_{1}, u_{2}, u_{3}\right) \text {. }
$$

Also let

$$
\begin{aligned}
& \epsilon_{2}=\epsilon_{2}(h)=u^{\prime}\left(x_{1}+h ; x_{1}+h, x_{2}, x_{3} ; u_{1}, u_{2}, u_{3}\right)-\beta_{2}, \text { and } \\
& \epsilon_{3}=\epsilon_{3}(h)=u^{\prime \prime}\left(x_{1}+h ; x_{1}+h, x_{2}, x_{3}, u_{1}, u_{2}, u_{3}\right)-\beta_{3} .
\end{aligned}
$$

By Theorem 3.1, $\epsilon_{2}=\epsilon_{2}(h) \rightarrow 0$ and $\epsilon_{3}=\epsilon_{3}(h) \rightarrow 0$, as $h \rightarrow 0$. As in part (a), let $y\left(x ; p, c_{1}, c_{2}, c_{3}\right)$ denote the solution of (1) satisfying the initial conditions

$$
y(p)=c_{1}, y^{\prime}(p)=c_{2}, y^{\prime \prime}(p)=c_{3},
$$

where $p \in(a, b)$ and $c_{1}, c_{2}, c_{3} \in \mathbb{R}$.

Then, viewing the solutions $u$ as solutions of initial value problems and using a telescoping sum,

$$
\begin{aligned}
z_{1 h}(x)= & \frac{1}{h}\left\{u\left(x ; x_{1}+h, x_{2}, x_{3}, u_{1}, u_{2}, u_{3}\right)-u\left(x ; x_{1}, x_{2}, x_{3}, u_{1}, u_{2}, u_{3}\right)\right\} \\
= & \frac{1}{h}\left\{y\left(x ; x_{1}+h, u_{1}, \beta_{2}+\epsilon_{2}, \beta_{3}+\epsilon_{3}\right)-y\left(x ; x_{1}, u_{1}, \beta_{2}, \beta_{3}\right)\right\} \\
= & \frac{1}{h}\left\{\left[y\left(x ; x_{1}+h, u_{1}, \beta_{2}+\epsilon_{2}, \beta_{3}+\epsilon_{3}\right)-y\left(x ; x_{1}, u_{1}, \beta_{2}+\epsilon_{2}, \beta_{3}+\epsilon_{3}\right)\right]\right. \\
& +\left[y\left(x ; x_{1}, u_{1}, \beta_{2}+\epsilon_{2}, \beta_{3}+\epsilon_{3}\right)-y\left(x ; x_{1}, u_{1}, \beta_{2}, \beta_{3}+\epsilon_{3}\right)\right] \\
& \left.+\left[y\left(x ; x_{1}, u_{1}, \beta_{2}, \beta_{3}+\epsilon_{3}\right)-y\left(x ; x_{1}, u_{1}, \beta_{2}, \beta_{3}\right)\right]\right\} .
\end{aligned}
$$


By Theorem 2.1 and the Mean Value Theorem,

$$
\begin{aligned}
z_{1 h}(x)= & \frac{1}{h} \beta\left(x ; y\left(x ; x_{1}+\bar{h}, u_{1}, \beta_{2}+\epsilon_{2}, \beta_{3}+\epsilon_{3}\right)\right)\left(x_{1}+h-x_{1}\right) \\
& +\frac{1}{h} \alpha_{2}\left(x ; y\left(x ; x_{1}, u_{1}, \beta_{2}+\bar{\epsilon}_{2}, \beta_{3}+\epsilon_{3}\right)\right)\left(\beta_{2}+\epsilon_{2}-\beta_{2}\right) \\
& +\frac{1}{h} \alpha_{3}\left(x ; y\left(x ; x_{1}, u_{1}, \beta_{2}, \beta_{3}+\bar{\epsilon}_{3}\right)\right)\left(\beta_{3}+\epsilon_{3}-\beta_{3}\right),
\end{aligned}
$$

where $\bar{h}$ is between 0 and $h, \bar{\epsilon}_{2}$ is between 0 and $\epsilon_{2}, \bar{\epsilon}_{3}$ is between 0 and $\epsilon_{3}$, and $\beta(x ; y(\cdot))$ is the solution of the variational equation (2) along $y\left(x ; x_{1}+\bar{h}, u_{1}, \beta_{2}+\epsilon_{2}, \beta_{3}+\epsilon_{3}\right)$ and satisfies the initial conditions $\beta^{(i)}\left(x_{1}+\bar{h}\right)=-y^{(i+1)}\left(x_{1}+\bar{h}\right), i=0,1,2$, and where $\alpha_{2}(x ; y(\cdot))$ and $\alpha_{3}(x ; y(\cdot))$ are solutions of $(2)$ along $y(\cdot)$, and satisfy the initial conditions

$$
\begin{aligned}
& \alpha_{2}\left(x_{1}\right)=0, \alpha_{2}^{\prime}\left(x_{1}\right)=1, \alpha^{\prime \prime}\left(x_{1}\right)=0, \\
& \alpha_{3}\left(x_{1}\right)=0, \alpha_{3}^{\prime}\left(x_{1}\right)=0, \alpha_{3}^{\prime \prime}\left(x_{1}\right)=1 .
\end{aligned}
$$

By simplifying the last expression for $z_{1 h}(x)$, we have

$$
\begin{aligned}
z_{1 h}(x)= & \beta\left(x ; y\left(x ; x_{1}+\bar{h}, u_{1}, \beta_{2}+\epsilon_{2}, \beta_{3}+\epsilon_{3}\right)\right) \\
& +\frac{\epsilon_{2}}{h} \alpha_{2}\left(x ; y\left(x ; x_{1}, u_{1}, \beta_{2}+\bar{\epsilon}_{2}, \beta_{3}+\epsilon_{3}\right)\right) \\
& +\frac{\epsilon_{3}}{h} \alpha_{3}\left(x ; y\left(x ; x_{1}, u_{1}, \beta_{2}, \beta_{3}+\bar{\epsilon}_{3}\right)\right) .
\end{aligned}
$$

To show that $\lim _{h \rightarrow 0} z_{1 h}$ exists, it suffices to show that $\lim _{h \rightarrow 0} \frac{\epsilon_{2}}{h}$ and $\lim _{h \rightarrow 0} \frac{\epsilon_{3}}{h}$ exist.

Recall that $z_{1 h}^{\prime}\left(x_{2}\right)=0$ and $z_{1 h}^{\prime}\left(x_{3}\right)=0$, for every $h \neq 0$. Using this fact, we obtain two equations in two unknowns:

$$
\begin{aligned}
& -\beta^{\prime}\left(x_{2} ; y\left(x ; x_{1}+\bar{h}, u_{1}, \beta_{2}+\epsilon_{2}, \beta_{3}+\epsilon_{3}\right)\right) \\
= & \frac{\epsilon_{2}}{h} \alpha_{2}^{\prime}\left(x_{2} ; y\left(x ; x_{1}, u_{1}, \beta_{2}+\bar{\epsilon}_{2}, \beta_{3}+\epsilon_{3}\right)\right)+\frac{\epsilon_{3}}{h} \alpha_{3}^{\prime}\left(x_{2} ; y\left(x ; x_{1}, u_{1}, \beta_{2}, \beta_{3}+\bar{\epsilon}_{3}\right)\right), \\
& -\beta^{\prime}\left(x_{3} ; y\left(x ; x_{1}+\bar{h}, u_{1}, \beta_{2}+\epsilon_{2}, \beta_{3}+\epsilon_{3}\right)\right) \\
= & \frac{\epsilon_{2}}{h} \alpha_{2}^{\prime}\left(x_{3} ; y\left(x ; x_{1}, u_{1}, \beta_{2}+\bar{\epsilon}_{2}, \beta_{3}+\epsilon_{3}\right)\right)+\frac{\epsilon_{3}}{h} \alpha_{3}^{\prime}\left(x_{3} ; y\left(x ; x_{1}, u_{1}, \beta_{2}, \beta_{3}+\bar{\epsilon}_{3}\right)\right) .
\end{aligned}
$$

We can determine $\frac{\epsilon_{2}}{h}$ and $\frac{\epsilon_{3}}{h}$ from Cramer's Rule by

$$
\begin{gathered}
\frac{\epsilon_{2}}{h}=\frac{\left|\begin{array}{ll}
-\beta^{\prime}\left(x_{2} ; y(\cdot)\right) & \alpha_{3}^{\prime}\left(x_{2} ; y(\cdot)\right) \\
-\beta^{\prime}\left(x_{3} ; y(\cdot)\right) & \alpha_{3}^{\prime}\left(x_{3} ; y(\cdot)\right)
\end{array}\right|}{\left|\begin{array}{ll}
\alpha_{2}^{\prime}\left(x_{2} ; y(\cdot)\right) & \alpha_{3}^{\prime}\left(x_{2} ; y(\cdot)\right) \\
\alpha_{2}^{\prime}\left(x_{3} ; y(\cdot)\right) & \alpha_{3}^{\prime}\left(x_{3} ; y(\cdot)\right)
\end{array}\right|} \equiv \frac{D_{2}(h)}{D(h)}, \text { and } \\
\frac{\epsilon_{3}}{h}=\frac{\left|\begin{array}{ll}
\alpha_{2}^{\prime}\left(x_{2} ; y(\cdot)\right) & -\beta^{\prime}\left(x_{2} ; y(\cdot)\right) \\
\alpha_{2}^{\prime}\left(x_{3} ; y(\cdot)\right) & -\beta^{\prime}\left(x_{3} ; y(\cdot)\right)
\end{array}\right|}{D(h)} \equiv \frac{D_{3}(h)}{D(h)},
\end{gathered}
$$


provided $D(h) \neq 0$, and where $y(\cdot)$ denotes the solutions of $(1)$ from above, and again we are suppressing the variables. Now, $\alpha_{2}\left(x ; y\left(x ; x_{1}, u_{1}, \beta_{2}, \beta_{2}\right)\right)$ and $\alpha_{3}\left(x ; y\left(x ; x_{1}, u_{1}, \beta_{2}, \beta_{3}\right.\right.$ )) are both zero at $x=x_{1}$, and as a consequence any linear combination is zero at $x=x_{1}$. It folows from (iii') that no nontrivial linear combination $A \alpha_{2}\left(x ; y\left(x ; x_{1}, u_{1}, \beta_{2}, \beta_{3}\right)\right)+$ $B \alpha_{3}\left(x ; y\left(x ; x_{1}, u_{1}, \beta_{2}, \beta_{3}\right)\right)$ has derivative equal to 0 at $x=x_{2}$ and $x=x_{3}$. This is equivalent to

$$
D=\left|\begin{array}{cc}
\alpha_{2}^{\prime}\left(x_{2} ; y\left(x ; x_{1}, u_{1}, \beta_{2}, \beta_{3}\right)\right) & \alpha_{3}^{\prime}\left(x_{2} ; y\left(x ; x_{1}, u_{1}, \beta_{2}, \beta_{3}\right)\right) \\
\alpha_{2}^{\prime}\left(x_{3} ; y\left(x ; x_{1}, u_{1}, \beta_{2}, \beta_{3}\right)\right) & \alpha_{3}^{\prime}\left(x_{3} ; y\left(x ; x_{1}, u_{1}, \beta_{2}, \beta_{3}\right)\right)
\end{array}\right| \neq 0
$$

By Theorem 3.1, $D(h) \neq 0$ for $h$ sufficiently small. In fact, $\lim _{h \rightarrow 0} D(h)=D$. This implies $\lim _{h \rightarrow 0} \frac{\epsilon_{2}(h)}{h}=\lim _{h \rightarrow 0} \frac{D_{2}(h)}{D(h)} \equiv \frac{D_{2}}{D} \equiv$ constant, and $\lim _{h \rightarrow 0} \frac{\epsilon_{3}}{h}=\lim _{h \rightarrow 0} \frac{D_{3}(h)}{D(h)} \equiv \frac{D_{3}}{D} \equiv$ constant. Returning to $z_{1 h}(x)$, we now have

$$
\begin{aligned}
z_{1 h}(x)= & \beta\left(x ; y\left(x ; x_{1}+\bar{h}, u_{1}, \beta_{2}+\epsilon_{2}, \beta_{3}+\epsilon_{3}\right)\right) \\
& +\frac{D_{2}(h)}{D(h)} \alpha_{2}\left(x ; y\left(x ; x_{1}, u_{1}, \beta_{2}+\bar{\epsilon}_{2}, \beta_{3}+\epsilon_{3}\right)\right) \\
& +\frac{D_{3}(h)}{D(h)} \alpha_{3}\left(x ; y\left(x ; x_{1}, u_{1}, \beta_{2}, \beta_{3}+\overline{\epsilon_{3}}\right)\right),
\end{aligned}
$$

and hence $\lim _{h \rightarrow 0} z_{1 h}(x)$ exists. Let $\lim _{h \rightarrow 0} z_{1 h}(x) \equiv z_{1}(x)$. In particular,

$$
z_{1}(x)=\frac{\partial}{\partial x_{1}} u\left(x ; x_{1}, x_{2}, x_{3}, u_{1}, u_{2}, u_{3}\right)
$$

because of the construction of $z_{1 h}(x)$. Since

$$
\begin{aligned}
z_{1}(x)= & \lim _{h \rightarrow 0} z_{1 h}(x) \\
= & \beta\left(x ; y\left(x ; x_{1}, u_{1}, \beta_{2}, \beta_{3}\right)\right)+\frac{D_{2}}{D} \alpha_{2}\left(x ; y\left(x ; x_{1}, u_{1}, \beta_{2}, \beta_{3}\right)\right) \\
& +\frac{D_{3}}{D} \alpha_{3}\left(x ; y\left(x ; x_{1}, u_{1}, \beta_{2}, \beta_{3}\right)\right),
\end{aligned}
$$

we have $z_{1}(x)$ is the solution of the variational equation (2) along $y(x)=y\left(x ; x_{1}, u_{1}, \beta_{2}\right.$, $\beta_{3}$ ) because $\beta, \alpha_{2}, \alpha_{3}$ are solutions of (2) along $y(x)$. Further,

$$
\begin{aligned}
z_{1}\left(x_{1}\right) & =\beta\left(x_{1} ; y(x)\right)+\frac{D_{2}}{D} \alpha_{2}\left(x_{1} ; y(x)\right)+\frac{D_{3}}{D} \alpha_{3}\left(x_{1} ; y(x)\right) \\
& =-y^{\prime}\left(x_{1}\right)+\frac{D_{2}}{D}(0)+\frac{D_{3}}{D}(0) \\
& =-u^{\prime}\left(x_{1}\right) .
\end{aligned}
$$


And again from an earlier part of this proof,

$$
\begin{aligned}
& z_{1}^{\prime}\left(x_{2}\right)=\lim _{h \rightarrow 0} z_{1 h}^{\prime}\left(x_{2}\right)=\lim _{h \rightarrow 0} 0=0, \\
& z_{1}^{\prime}\left(x_{3}\right)=\lim _{h \rightarrow 0} z_{1 h}^{\prime}\left(x_{3}\right)=\lim _{h \rightarrow 0} 0=0 .
\end{aligned}
$$

Note that above we use $y\left(x ; x_{1}, u_{1}, \beta_{2}, \beta_{3}\right)=u\left(x ; x_{1}, x_{2}, x_{3}, u_{1}, u_{2}, u_{3}\right)$.

So, $z_{1}(x)=\frac{\partial}{\partial x_{1}} u\left(x ; x_{1}, x_{2}, x_{3}, u_{1}, u_{2}, u_{3}\right)$ is the solution of the variational equation (2) along $u(x)=u\left(x ; x_{1}, x_{2}, x_{3}, u_{1}, u_{2}, u_{3}\right)$ and satisfies

$$
\begin{aligned}
& z_{1}\left(x_{1}\right)=-u\left(x_{1}\right) \\
& z_{1}^{\prime}\left(x_{2}\right)=0 \\
& z_{1}^{\prime}\left(x_{3}\right)=0 .
\end{aligned}
$$

For the next part of (b), we show that $\frac{\partial u}{\partial x_{2}}$ exists on $(\alpha, \omega)$, and that $z_{2} \equiv \frac{\partial u}{\partial x_{2}}$ is the solution of the variational equation (2) along $u(x)$ and satisfies the boundary conditions

$$
z_{2}\left(x_{1}\right)=0, z_{2}^{\prime}\left(x_{2}\right)=-u^{\prime \prime}\left(x_{2}\right), z_{2}^{\prime}\left(x_{3}\right)=0 .
$$

Let $\delta>0$ be given as in Theorem 3.1. Choose $h \neq 0$ with $|h|<\delta$ and define

$$
z_{2 h}(x)=\frac{u\left(x ; x_{1}, x_{2}+h, x_{3}, u_{1}, u_{2}, u_{3}\right)-u\left(x ; x_{1}, x_{2}, x_{3}, u_{1}, u_{2}, u_{3}\right)}{h} .
$$

Note that $z_{2 h}\left(x_{1}\right)=\frac{u_{1}-u_{1}}{h}=0$, for every $h \neq 0$, and $z_{2 h}^{\prime}\left(x_{3}\right)=\frac{u_{3}-u_{3}}{h}=0$, for every $h \neq 0$. Let

$$
\begin{aligned}
& \beta_{1}=u\left(x_{2} ; x_{1}, x_{2}, x_{3}, u_{1}, u_{2}, u_{3}\right), \text { and } \\
& \beta_{3}=u^{\prime \prime}\left(x_{2} ; x_{1}, x_{2}, x_{3}, u_{1}, u_{2}, u_{3}\right) .
\end{aligned}
$$

Also let $\epsilon_{1}=\epsilon_{1}(h)=u\left(x_{2}+h ; x_{1}, x_{2}+h, x_{3}, u_{1}, u_{2}, u_{3}\right)-\beta_{1}$, and

$$
\epsilon_{3}=\epsilon_{3}(h)=u^{\prime \prime}\left(x_{2}+h ; x_{1}, x_{2}+h, x_{3}, u_{1}, u_{2}, u_{3}\right)-\beta_{3} .
$$

By Theorem 3.1, $\epsilon_{1}=\epsilon_{1}(h) \rightarrow 0$ and $\epsilon_{3}=\epsilon_{3}(h) \rightarrow 0$, as $h \rightarrow 0$.

Now, given $p \in(a, b)$ and $c_{1}, c_{2}, c_{3} \in \mathbb{R}$, let $y(x)=y\left(x ; p, c_{1}, c_{2}, c_{3}\right)$ denote the solution of (1) satisfying the initial conditions

$$
y(p)=c_{1}, y^{\prime}(p)=c_{2}, \text { and } y^{\prime \prime}(p)=c_{3} .
$$

Again, viewing the solutions $u$ as solutions of initial value problems and using a telescoping sum,

$$
\begin{aligned}
z_{2 h}(x)= & \frac{1}{h}\left\{u\left(x ; x_{1}, x_{2}+h, x_{3}, u_{1}, u_{2}, u_{3}\right)-u\left(x ; x_{1}, x_{2}, x_{3}, u_{1}, u_{2}, u_{3}\right)\right\} \\
= & \frac{1}{h}\left\{y\left(x ; x_{2}+h, \beta_{1}+\epsilon_{1}, u_{2}, \beta_{3}+\epsilon_{3}\right)-y\left(x ; x_{2}, \beta_{1}, u_{2}, \beta_{3}\right)\right\} \\
= & \frac{1}{h}\left\{\left[y\left(x ; x_{2}+h, \beta_{1}+\epsilon_{1}, u_{2}, \beta_{3}+\epsilon_{3}\right)-y\left(x ; x_{2}, \beta_{1}+\epsilon_{1}, u_{2}, \beta_{3}+\epsilon_{3}\right)\right]\right. \\
& +\frac{1}{h}\left[y\left(x ; x_{2}, \beta_{1}+\epsilon_{1}, u_{2}, \beta_{3}+\epsilon_{3}\right)-y\left(x ; x_{2}, \beta_{1}, u_{2}, \beta_{3}+\epsilon_{3}\right)\right] \\
& +\frac{1}{h}\left[y\left(x ; x_{2}, \beta_{1}, u_{2}, \beta_{3}+\epsilon_{3}\right)-y\left(x ; x_{2}, \beta_{1}, u_{2}, \beta_{3}\right)\right\} .
\end{aligned}
$$


By Theorem 2.1 and the Mean Value Theorem,

$$
\begin{aligned}
z_{2 h}(x)= & \frac{1}{h} \beta\left(x ; y\left(x ; x_{2}+\bar{h}, \beta_{1}+\epsilon_{1}, u_{2}, \beta_{3}+\epsilon_{3}\right)\right)\left(x_{2}+h-x_{2}\right) \\
& +\frac{1}{h} \alpha_{1}\left(x ; y\left(x ; x_{2}, \beta_{1}+\bar{\epsilon}_{1}, u_{2}, \beta_{3}+\epsilon_{3}\right)\right)\left(\beta_{1}+\epsilon_{1}-\beta_{1}\right) \\
& +\frac{1}{h} \alpha_{3}\left(x ; y\left(x ; x_{2}, \beta_{1}, u_{2}, \beta_{3}+\bar{\epsilon}_{3}\right)\right)\left(\beta_{3}+\epsilon_{3}-\beta_{3}\right),
\end{aligned}
$$

where $\beta(x ; y(\cdot))$ is a solution of the variational equation (2) along the solution $y(\cdot)$ of $(1)$ and satisfies the initial conditions

$$
\begin{aligned}
\beta\left(x_{2}+\bar{h}\right) & =-y^{\prime}\left(x_{2}+\bar{h}\right) \\
\beta^{\prime}\left(x_{2}+\bar{h}\right) & =-y^{\prime \prime}\left(x_{2}+\bar{h}\right) \\
\beta^{\prime \prime}\left(x_{2}+\bar{h}\right) & =-y^{\prime \prime \prime}\left(x_{2}+\bar{h}\right) .
\end{aligned}
$$

Also, $\alpha_{1}(x ; y(\cdot))$ and $\alpha_{3}(x ; y(\cdot))$ are solutions to the variational equation (2) along $y(\cdot)$ of (1) and satisfy the initial conditions

$$
\begin{aligned}
& \alpha_{1}\left(x_{2}\right)=1, \alpha_{1}^{\prime}\left(x_{2}\right)=0, \alpha_{1}^{\prime \prime}\left(x_{2}\right)=0, \\
& \alpha_{3}\left(x_{2}\right)=0, \alpha_{3}^{\prime}\left(x_{2}\right)=0, \alpha_{3}^{\prime \prime}\left(x_{2}\right)=1,
\end{aligned}
$$

where $\bar{h}$ is between 0 and $h, \bar{\epsilon}_{i}$ is between 0 and $\epsilon_{i}, i=1,3$. So,

$$
\begin{aligned}
z_{2 h}(x)= & \beta\left(x ; y\left(x ; x_{2}+\bar{h}, \beta_{1}+\epsilon_{1}, u_{2}, \beta_{3}+\epsilon_{3}\right)\right)+\frac{\epsilon_{1}}{h} \alpha_{1}\left(x ; y\left(x ; x_{2}, \beta_{1}+\bar{\epsilon}_{1}, u_{2}, \beta_{3}+\epsilon_{3}\right)\right) \\
& +\frac{\epsilon_{3}}{h} \alpha_{3}\left(x ; y\left(x ; x_{2}, \beta_{1}, u_{2}, \beta_{3}+\bar{\epsilon}_{3}\right)\right) .
\end{aligned}
$$

To show that $\lim _{h \rightarrow 0} z_{2 h}(x)$ exists, it again suffices to show that $\lim _{h \rightarrow 0} \frac{\epsilon_{1}}{h}$ and $\lim _{h \rightarrow 0} \frac{\varepsilon_{3}}{h}$ exist. Recall that $z_{2 h}\left(x_{1}\right)=z_{2 h}^{\prime}\left(x_{3}\right)=0$, for every $h \neq 0$. This gives the system of two equations in two unknowns:

$$
\begin{aligned}
-\beta\left(x_{1} ; y\left(x ; x_{2}+\bar{h}, \beta_{1}+\epsilon_{1}, u_{2}, \beta_{3}+\epsilon_{3}\right)\right)= & \frac{\epsilon_{1}}{h} \alpha_{1}\left(x_{1} ; y\left(x ; x_{2}, \beta_{1}+\overline{\epsilon_{1}}, u_{2}, \beta_{3}+\epsilon_{3}\right)\right) \\
& +\frac{\epsilon_{3}}{h} \alpha_{3}\left(x_{1} ; y\left(x ; x_{2}, \beta_{1}, u_{2}, \beta_{3}+\bar{\epsilon}_{3}\right)\right), \text { and } \\
-\beta^{\prime}\left(x_{3} ; y\left(x ; x_{2}+\bar{h}, \beta_{1}+\epsilon_{1}, u_{2}, \beta_{3}+\epsilon_{3}\right)\right)= & \frac{\epsilon_{1}}{h} \alpha_{1}^{\prime}\left(x_{3} ; y\left(x ; x_{2}, \beta_{1}+\bar{\epsilon}_{1}, u_{2}, \beta_{3}+\epsilon_{3}\right)\right) \\
& +\frac{\epsilon_{3}}{h} \alpha_{3}^{\prime}\left(x_{3} ; y\left(x ; x_{2}, \beta_{1}, u_{2}, \beta_{3}+\bar{\epsilon}_{3}\right)\right) .
\end{aligned}
$$

Again, using Cramer's Rule, we solve for $\frac{\epsilon_{1}}{h}$ and $\frac{\epsilon_{3}}{h}$ by

$$
\frac{\epsilon_{1}}{h}=\frac{\left|\begin{array}{ll}
-\beta\left(x_{1} ; y(\cdot)\right) & \alpha_{3}\left(x_{1} ; y(\cdot)\right) \\
-\beta^{\prime}\left(x_{3} ; y(\cdot)\right) & \alpha_{3}^{\prime}\left(x_{3} ; y(\cdot)\right)
\end{array}\right|}{\left|\begin{array}{ll}
\alpha_{1}\left(x_{1} ; y(\cdot)\right) & \alpha_{3}\left(x_{1} ; y(\cdot)\right) \\
\alpha_{1}^{\prime}\left(x_{3} ; y(\cdot)\right) & \alpha_{3}^{\prime}\left(x_{3} ; y(\cdot)\right)
\end{array}\right|} \equiv \frac{D_{1}(h)}{D(h)},
$$


and

$$
\frac{\epsilon_{3}}{h}=\frac{\left|\begin{array}{cc}
\alpha_{1}\left(x_{1} ; y(\cdot)\right) & -\beta\left(x_{1} ; y(\cdot)\right) \\
\alpha_{1}^{\prime}\left(x_{3} ; y(\cdot)\right) & -\beta^{\prime}\left(x_{3} ; y(\cdot)\right)
\end{array}\right|}{D(h)} \equiv \frac{D_{3}(h)}{D(h)}
$$

provided $D(h) \neq 0$. Here, $y(\cdot)$ denotes the solutions of $(1)$ with respect to their initial conditions as previously given.

The usual argument can be made that $D(h) \neq 0$, for $h$ small, and with $\lim _{h \rightarrow 0} D(h)$ $=D$, for the appropriate $D$, we have

$$
\begin{aligned}
& \lim _{h \rightarrow 0} \frac{\epsilon_{1}}{h}=\lim _{h \rightarrow 0} \frac{D_{1}(h)}{D(h)}=\frac{D_{1}}{D} \equiv \text { constant, and } \\
& \lim _{h \rightarrow 0} \frac{\epsilon_{3}}{h}=\lim _{h \rightarrow 0} \frac{D_{3}(h)}{D(h)}=\frac{D_{3}}{D} \equiv \text { constant } .
\end{aligned}
$$

This now gives us

$$
z_{2 h}(x)=\beta(x ; y(\cdot))+\frac{D_{1}(h)}{D(h)} \alpha(x ; y(\cdot))+\frac{D_{3}(h)}{D(h)} \alpha_{3}(x ; y(\cdot))
$$

and so $\lim _{h \rightarrow 0} z_{2 h}(x)$ exists.

Further, because of the definition of $z_{2 h}(x)$,

$$
\lim _{h \rightarrow 0} z_{2 h}(x)=\frac{\partial u}{\partial x_{2}}\left(x ; x_{1}, x_{2}, x_{3}, u_{1}, u_{2}, u_{3}\right) \equiv z_{2}(x)
$$

That is,

$$
\begin{aligned}
z_{2}(x)= & \beta\left(x ; y\left(x ; x_{2}, \beta_{1}, u_{2}, \beta_{3}\right)\right)+\frac{D_{1}}{D} \alpha_{1}\left(x ; y\left(x ; x_{2}, \beta_{1}, u_{2}, \beta_{3}\right)\right) \\
& +\frac{D_{3}}{D} \alpha_{3}\left(x ; y\left(x ; x_{2}, \beta_{1}, u_{2}, \beta_{3}\right)\right) .
\end{aligned}
$$

Hence, $z_{2}(x)$ is a solution to the variational equation (2) along $y\left(x ; x_{2}, \beta_{1}, u_{2}, \beta_{3}\right)=$ $u\left(x ; x_{1}, x_{2}, x_{3}, u_{1}, u_{2}, u_{3}\right)$, because $\beta, \alpha_{1}$, and $\alpha_{3}$ are.

Moreover,

$$
\begin{aligned}
& z_{2}\left(x_{1}\right)=\lim _{h \rightarrow 0} z_{2 h}\left(x_{1}\right)=\lim _{h \rightarrow 0} 0=0, \\
& z_{2}^{\prime}\left(x_{2}\right)=\beta^{\prime}\left(x_{2} ; y\left(x ; x_{2}, \beta_{1}, u_{2}, \beta_{3}\right)\right)=-y^{\prime \prime}\left(x_{2} ; x_{2}, \beta_{1}, u_{2}, \beta_{3}\right) \\
& =-u^{\prime \prime}\left(x_{2} ; x_{1}, x_{2}, x_{3}, u_{1}, u_{2}, u_{3}\right), \text { and } \\
& z_{2}^{\prime}\left(x_{3}\right)=\lim _{h \rightarrow 0} z_{2 h}^{\prime}\left(x_{3}\right)=\lim _{h \rightarrow 0} 0=0 .
\end{aligned}
$$

This completes the arguments for $\frac{\partial u}{\partial x_{2}}$. As remarked earlier in this proof, the arguments for $\frac{\partial u}{\partial x_{3}}$ are completely analogous. This completes (b). 
For part (c) of the theorem, assume that parts (a) and (b) are satisfied. In particular, we note that $z_{1}(x), z_{2}(x), z_{3}(x), y_{1}(x), y_{2}(x)$, and $y_{3}(x)$ are all solutions of the same variational equation (2). Now, observe the boundary conditions that $z_{1}(x)$ and $y_{1}(x)$ satisfy:

$$
\begin{aligned}
& z_{1}\left(x_{1}\right)=-u^{\prime}\left(x_{1}\right)(1)=-u^{\prime}\left(x_{1}\right) y_{1}\left(x_{1}\right) \\
& z_{1}^{\prime}\left(x_{2}\right)=-u^{\prime}\left(x_{1}\right)(0)=-u^{\prime}\left(x_{1}\right) y_{1}^{\prime}\left(x_{2}\right) \\
& z_{1}^{\prime}\left(x_{3}\right)=-u^{\prime}\left(x_{1}\right)(0)=-u^{\prime}\left(x_{1}\right) y_{1}^{\prime}\left(x_{3}\right) .
\end{aligned}
$$

Hence, by (iii'), $z_{1}(x) \equiv-u^{\prime}\left(x_{1}\right) y_{1}(x)$, or specifically, $\frac{\partial u}{\partial x_{1}}(x)=-u^{\prime}\left(x_{1}\right) \frac{\partial u}{\partial u_{1}}(x)$.

Similarly, $z_{2}(x)$ and $y_{2}(x)$ satisfy the boundary conditions,

$$
\begin{aligned}
& z_{2}^{\prime}\left(x_{1}\right)=-u^{\prime \prime}\left(x_{2}\right)(0)=-u^{\prime \prime}\left(x_{2}\right) y_{2}\left(x_{1}\right), \\
& z_{2}^{\prime}\left(x_{2}\right)=-u^{\prime \prime}\left(x_{2}\right)(1)=-u^{\prime \prime}\left(x_{2}\right) y_{2}^{\prime}\left(x_{2}\right), \\
& z_{2}^{\prime}\left(x_{3}\right)=-u^{\prime \prime}\left(x_{2}\right)(0)=-u^{\prime \prime}\left(x_{2}\right) y_{2}^{\prime}\left(x_{3}\right) .
\end{aligned}
$$

Again, by (iii'), $z_{2}(x) \equiv-u^{\prime \prime}\left(x_{2}\right) y_{2}(x)$, or equivalently, $\frac{\partial u}{\partial x_{2}}(x)=-u^{\prime \prime}\left(x_{2}\right) \frac{\partial u}{\partial u_{2}}(x)$.

Finally,

$$
\begin{aligned}
& z_{3}\left(x_{1}\right)=-u^{\prime \prime}\left(x_{3}\right)(0)=-u^{\prime \prime}\left(x_{3}\right) y_{3}\left(x_{1}\right), \\
& z_{3}^{\prime}\left(x_{2}\right)=-u^{\prime \prime}\left(x_{3}\right)(0)=-u^{\prime \prime}\left(x_{3}\right) y_{3}^{\prime}\left(x_{2}\right), \\
& z_{3}^{\prime}\left(x_{3}\right)=-u^{\prime \prime}\left(x_{3}\right)(1)=-u^{\prime \prime}\left(x_{3}\right) y_{3}^{\prime}\left(x_{3}\right),
\end{aligned}
$$

which by (iii'), gives us $\frac{\partial u}{\partial x_{3}}(x)=-u^{\prime \prime}\left(x_{3}\right) \frac{\partial u}{\partial u_{3}}(x)$. This completes the proof.

\section{Other Analogues of Peano's Theorem}

For the BVP's (1), (4), we state, without proof, first a theorem dealing with continuous dependence on boundary conditions, followed by a theorem concerning differentiation with respect to boundary conditions. Proof of the theorem on continuous dependence follows along the lines in [7], and the proof of the differentiation theorem closely follows that of Theorem 3.1.

Theorem 4.1 Assume that (i), (ii), and (iv) are satisfied with respect to (1). Let $u(x)$ be a solution of $(1)$ with maximal interval of existence $(\alpha, \omega) \subseteq(a, b)$ and let $\alpha<$ $x_{0}<x_{1}<x_{2}<x_{3}<\omega$ be given. Then there exists a $\delta>0$ such that, for $\left|x_{1}-t_{1}\right|<$ $\delta,\left|x_{2}-t_{2}\right|<\delta,\left|u\left(x_{1}\right)-y_{1}\right|<\delta,\left|u^{\prime}\left(x_{1}\right)-y_{2}\right|<\delta$, and $\left|u^{\prime}\left(x_{2}\right)-y_{3}\right|<\delta$, there is a unique solution $u_{\delta}(x)$ of (1) whose maximal interval of existence is $\left(\alpha_{\delta}, \omega_{\delta}\right) \supset\left[x_{0}, x_{3}\right]$, and is such that $u_{\delta}\left(t_{1}\right)=y_{1}, u_{\delta}^{\prime}\left(t_{1}\right)=y_{2}, u_{\delta}^{\prime}\left(t_{2}\right)=y_{3}$. Moreover, $\left\{u_{\delta}^{(j)}(x)\right\}$ converges uniformly to $u^{(j)}(x)$ as $\delta \rightarrow 0$ on $\left[x_{0}, x_{3}\right]$, for each $j=0,1,2$.

Theorem 4.2 Assume (i), (ii), and (iv) are satisfied. Also assume that the variational equation (2) along all solutions $y(x)$ of (1) satisfies $\left(i v^{\prime}\right)$ on $(a, b)$. Let $u(x)$ be a solution of (1) with maximal interval of existence $(\alpha, \omega) \subseteq(a, b)$. Let $\alpha<x_{1}<x_{2}<\omega$ be given so that $u(x)=u\left(x ; x_{1}, x_{2}, u_{1}, u_{2}, u_{3}\right)$, where $u\left(x_{1}\right)=u_{1}, u^{\prime}\left(x_{1}\right)=u_{2}$, and $u^{\prime}\left(x_{2}\right)=u_{3}$. Then 
(a) $\frac{\partial u}{\partial u_{1}}, \frac{\partial u}{\partial u_{2}}, \frac{\partial u}{\partial u_{3}}$ exist on $(\alpha, \omega)$ and $y_{i} \equiv \frac{\partial u}{\partial u_{i}}, 1 \leq i \leq 3$, is the solution of (2) along $u(x)$ and satisfies the boundary conditions

$$
\begin{aligned}
& y_{1}\left(x_{1}\right)=1, y_{1}^{\prime}\left(x_{1}\right)=0, y_{1}^{\prime}\left(x_{2}\right)=0 \\
& y_{2}\left(x_{1}\right)=0, y_{2}^{\prime}\left(x_{1}\right)=1, y_{2}^{\prime}\left(x_{2}\right)=0 \\
& y_{3}\left(x_{1}\right)=0, y_{3}^{\prime}\left(x_{1}\right)=0, y_{3}^{\prime}\left(x_{2}\right)=1
\end{aligned}
$$

(b) $\frac{\partial u}{\partial x_{1}}, \frac{\partial u}{\partial x_{2}}$ exist on $(\alpha, \omega)$ and $z_{i} \equiv \frac{\partial u}{\partial x_{i}}, 1 \leq i \leq 2$, is the solution of (2) along $u(x)$ and satisfies the boundary conditions

$$
\begin{aligned}
& z_{1}\left(x_{1}\right)=-u^{\prime}\left(x_{1}\right), z_{1}^{\prime}\left(x_{1}\right)=-u^{\prime \prime}\left(x_{1}\right), z_{1}^{\prime}\left(x_{2}\right)=0 \\
& z_{2}\left(x_{1}\right)=0, z_{2}^{\prime}\left(x_{1}\right)=0, z_{2}^{\prime}\left(x_{2}\right)=-u^{\prime \prime}\left(x_{2}\right) .
\end{aligned}
$$

(c) The partial derivatives satisfy

$$
\frac{\partial u}{\partial x_{1}}(x)=-u^{\prime}\left(x_{1}\right) \frac{\partial u}{\partial u_{1}}(x)-u^{\prime \prime}\left(x_{1}\right) \frac{\partial u}{\partial u_{2}}(x), \text { and } \frac{\partial u}{\partial x_{2}}(x)=-u^{\prime \prime}\left(x_{2}\right) \frac{\partial u}{\partial u_{3}}(x) .
$$

We conclude this paper by stating further analogues of Theorems 3.1 and 3.2 for BVP's (1), (5).

Theorem 4.3. Assume (i), (ii), and (v) are satisfied with respect to (1). Let $u(x)$ be a solution of (1) with maximal interval of existence $(\alpha, \omega) \subseteq(a, b)$ and let $\alpha<x_{0}<x_{1}<$ $x_{2}<x_{3}<\omega$ be given. Then there exists a $\delta>0$ such that, for $\left|x_{1}-t_{1}\right|<\delta,\left|x_{2}-t_{2}\right|<$ $\delta,\left|u\left(x_{1}\right)-y_{1}\right|<\delta,\left|u^{\prime}\left(x_{2}\right)-y_{2}\right|<\delta$, and $\left|u^{\prime \prime}\left(x_{2}\right)-y_{3}\right|<\delta$, there is a unique solution $u_{\delta}(x)$ of (1) whose maximal interval of existence is $\left(\alpha_{\delta}, \omega_{\delta}\right) \supset\left[x_{0}, x_{3}\right]$ and is such that $u_{\delta}\left(t_{1}\right)=y_{1}, u_{\delta}^{\prime}\left(t_{2}\right)=y_{2}$, and $u^{\prime \prime}\left(t_{2}\right)=y_{3}$. Further, $\left\{u_{\delta}^{(j)}(x)\right\}$ converges uniformly to $u^{(j)}(x)$, as $\delta \rightarrow 0$ on $\left[x_{0}, x_{3}\right]$, for each $j=0,1,2$.

Theorem 4.4. Assume (i), (ii) and (v) are satisfied. Also assume that the variational equation (2), along any solution $y(x)$ of $(1)$, satisfies $\left(v^{\prime}\right)$ on $(a, b)$. Let $u(x)$ be a solution of (1) with maximal interval of existence $(\alpha, \omega) \subseteq(a, b)$. Let $\alpha<x_{1}<x_{2}<\omega$ be given, so that $u(x)=u\left(x ; x_{1}, x_{2}, u_{1}, u_{2}, u_{3}\right)$, where $u\left(x_{1}\right)=u_{1}, u^{\prime}\left(x_{2}\right)=u_{2}$, and $u^{\prime \prime}\left(2_{2}\right)=u_{3}$. Then

(a) $\frac{\partial u_{1}}{\partial u_{1}}, \frac{\partial u}{\partial u_{2}}, \frac{\partial u}{\partial u_{3}}$ exist on $(\alpha, \omega)$ and $y_{i} \equiv \frac{\partial u}{\partial u_{i}}, 1 \leq i \leq 3$, is the solution of (2) along $u(x)$ and satisfies the boundary conditions

$$
\begin{aligned}
& y_{1}\left(x_{1}\right)=1, y_{1}^{\prime}\left(x_{2}\right)=0, y_{1}^{\prime \prime}\left(x_{2}\right)=0, \\
& y_{2}\left(x_{1}\right)=0, y_{2}^{\prime}\left(x_{2}\right)=1, y_{2}^{\prime \prime}\left(x_{2}\right)=0, \\
& y_{3}\left(x_{1}\right)=0, y_{3}^{\prime}\left(x_{2}\right)=0, y_{3}^{\prime \prime}\left(x_{2}\right)=1 .
\end{aligned}
$$

(b) $\frac{\partial u}{\partial x_{1}}, \frac{\partial u}{\partial x_{2}}$ exist on $(\alpha, \omega)$ and $z_{i} \equiv \frac{\partial u}{\partial x_{i}}, 1 \leq i \leq 2$, is the solution of (2) along $u(x)$ and satisfies the boundary conditions

$$
\begin{aligned}
& z_{1}\left(x_{1}\right)=-u^{\prime}\left(x_{1}\right), z_{1}^{\prime}\left(x_{2}\right)=0, z_{1}^{\prime \prime}\left(x_{2}\right)=0, \\
& z_{2}\left(x_{1}\right)=0, z_{2}^{\prime}\left(x_{2}\right)=-u^{\prime \prime}\left(x_{2}\right), z_{2}^{\prime \prime}\left(x_{2}\right)=-u^{\prime \prime \prime}\left(x_{2}\right) .
\end{aligned}
$$


(c) The partial derivatives satisfy

$$
\frac{\partial u}{\partial x_{1}}(x)=-u^{\prime}\left(x_{1}\right) \frac{\partial u}{\partial u_{1}}(x) \text {, and } \frac{\partial u}{\partial x_{2}}(x)=-u^{\prime \prime}\left(x_{2}\right) \frac{\partial u}{\partial u_{2}}(x)-u^{\prime \prime \prime}\left(x_{2}\right) \frac{\partial u}{\partial u_{3}}(x) \text {. }
$$

\section{References}

[1] G. A. Bliss, Lectures on the Calculus of Variations, University of Chicago Press, Chicago, 1946.

[2] D. Hankerson, Boundary Value Problems for $n$-th order difference equations, Ph.D. dissertation, University of Nebraska, Lincoln, Nebraska, 1986.

[3] P. Hartman, Ordinary Differential Equations, Wiley, New York, 1964.

[4] J. Henderson, "Right focal point boundary value problems for ordinary differential equations and variational equations", J. Math. Anal. Appl: 98 (1984), 363-377.

[5] J. Henderson, "Right $\left(m_{1} ; \ldots ; m_{\ell}\right)$ focal boundary value problems for third-order differential equations", J. Math. Phys. Sci. 18 (1984), 405-413.

[6] J. Henderson, "Disconjugacy, disfocality, and differentiation with respect to boundary conditions", J. Math. Anal. Appl. 121 (1987), 1-9.

[7] L. Jackson, "Uniqueness of solutions of boundary value problems for ordinary' differential equar tions", SIAM J. Math. Appl. 24 (1973), 535-538.

[8] A. Peterson, "An expression for the first conjugate point for an $n t h$ order nonlinear differential equation", Proc. Amer. Math. Soc. 61 (1976), 300-304.

[9] A. Peterson, "Comparison theorems and existence theorems for ordinary differential equations", $J$. Math. Anal. Appl. 55 (1976), 773-784.

[10] A. Peterson, "Existence-uniqueness for ordinary differential equations", J. Math. Anal. Appl. 64 (1978), 166-172.

[11] A. Peterson, "Existence-uniqueness for focal-point boundary value problems", SIAM J. Math. Anal. 12 (1981), 173-185.

[12] A. Peterson, "Existence and uniqueness theorems for nonlinear difference equations", J. Math. Anal. Appl. 125 (1987), 185-191.

[13] J. Spencer, "Relations between boundary value functions for a nonlinear differential equation and its variational equations", Canad. Math. Bull. 18 (1975), 269-276.

Department of Algebra, Combinatorics, and Analysis Auburn University Auburn, Alabama 36849 USA. 